\title{
Appendice à la partie 1 : « Discours Rapporté » et "Représentation de Discours Autre » - questions de dénomination ${ }^{1}$
}

L'abandon, ici, de la dénomination consacrée de « discours rapporté » - toujours dominante dans les travaux sur ce champ et exclusive dans les dictionnaires, manuels ou instructions pédagogiques officielles - pour celle de « représentation de discours autre » se joue à plusieurs niveaux.

\section{Inadéquation de « discours rapporté » : réponses diverses.}

Au premier niveau cet abandon participe de la longue suite de critiques formulées à l'encontre du terme " discours rapporté » relativement à ce qu'il désigne. Ces critiques se concentrent sur deux points :

(a) d'une part sur le caractère factuel et antérieur d'un événement langagier susceptible d'être "rapporté $»^{2}-$ auquel fait écho l'exemplification stéréotypique en "il a dit (que) »-, là où le champ visé inclut toute la diversité des dires non réalisés : projetés, niés, interrogés, imaginés ${ }^{3}$...

(b) d'autre part sur le caractère de reproduction transparente parfois associé au terme « discours rapporté » (ou de « reported speech ») méconnaissant la dimension interprétative de l'opération.

$\mathrm{Au}$ constat, largement partagé, du caractère malheureux de cette terminologie, répondent deux stratégies discursives : conserver la dénomination communément reçue comme étiquetage («nom propre ») d'un champ, envisagé sans tenir compte des restrictions (a) et (b) attachées au sens du terme « rapporter »; ou bien proposer, au risque de l'inflation, des innovations terminologiques ${ }^{4}$. Le choix entre les deux est évidemment corrélé au genre du discours où il s'effectue - les travaux de recherche manifestant, dans leur rapport aux dénominations établies, une plus grande liberté que les textes à visée pédagogique ou de vulgarisation

$1 C f$. ci-dessous (Appendice à la partie IV) la question du fonctionnement de citer/citation.

2 Cf. l'emploi usuel de rapport : de stage, de mission, d'activité...

3 Cf. ci-dessus chap. 2.2.1.

4 Pour des éléments sur ces propositions de dénomination (chez Peytard, Genette, de Gaulmyn...) voir notamment Rosier (1999 : 44 sq.), Mochet (2003 : 166).

ว Open Access. () 2020 Jacqueline Authier-Revuz, published by De Gruyter. (cc)BY-NC-ND This work is licensed under the Creative Commons Attribution-NonCommercial-NoDerivatives 4.0 License. https://doi.org/10.1515/9783110641226-006 
(cours, manuels, encyclopédies...), tenus discursivement, sinon institutionnellement, à les respecter.

À titre d'exemple - sans souci d'exhaustivité - des deux types de « réponses », on peut évoquer la démarche innovatrice de Peytard (1982) et celle, pédagogiquement contrainte, de Authier-Revuz (1993a).

C'est ainsi par les deux propositions de distinguer discours relaté vs évoqué (référent factuel $v s$ non factuel) et de poser une tripartition rapporté-transposé-narrativisé que Peytard répond respectivement aux deux difficultés (a) et (b). En revanche, le second texte ${ }^{5}$ - présenté sous la rubrique «Pour l'agrégation » par la revue L'information grammaticale - affiche dans le titre « Repères dans le champ du discours rapporté » la dénomination établie, pour, dès les premières lignes, l'expliciter sur le mode :

[...] le champ du discours rapporté, c'est-à-dire des modes de représentation dans un discours d'un autre discours (1993a, I : 38).

Si présentes que soient ensuite, dans le cours du texte, les formulations en " représenter un autre discours », nettement dominantes par rapport à celles en « rapporter » ou DR, il n'y a pas de « destitution » d'une dénomination en faveur d'une autre : la différence est significative entre expliciter ce que recouvre une nomination reçue et nommer autrement ${ }^{6}$.

C'est pour ma part, à partir de 2001, dans un cadre non pédagogique, celui du colloque "Le discours rapporté dans tous ses états ${ }^{7}$, que je renonce aux reformulations explicatives de "discours rapporté » pour une re-nomination en « représentation de discours autre » (RDA) affichée dans le titre et explicitée dans l'introduction ${ }^{8}$.

5 Rédigé à l'intention des étudiants «à distance » de la licence de lettres modernes de l'université Paris 3.

6 C'est ce que manifeste nettement, dans le volume Une langue : le français, Tomassone (2001) (dir.), collection « Grands repères culturels » de Hachette Education, le compromis entre l'auteur (Authier-Revuz 2001b) des pages 192-201 consacrées à ce champ, ouvrant celles-ci par le titre « La représentation du discours autre », aussitôt explicité en introduction quant à son rapport avec « discours rapporté », et les responsables éditoriaux - renvoyant, dans la table des matières, par le titre « Le discours rapporté », à ce même groupe de pages.

7 Premier colloque du groupe Ci-dit, à Bruxelles, $c f$. López-Muñoz et al. (2004).

8 Authier-Revuz (2004a : 35). 


\section{De « rapporté »à « représenté »}

Cette renomination en RDA du champ du « discours rapporté » n'est pas isolée : le paradigme de la "représentation » apparaît dans des travaux d'inspirations diverses et argumenté diversement depuis la fin des années1980․ Et ce mouvement est suffisamment marqué pour que, en 2002, le Dictionnaire d'analyse du discours (Charaudeau et Maingueneau) en fasse mention à l'article « Discours rapporté $»^{10}$. Mais le recours au terme, plus général, de "représenter » correspond à deux démarches différentes : l'une (1) substitue « représenter » à « rapporter », l'autre (2) inclut « rapporter » dans « représenter ».

\subsection{Remplacer « rapporter » par « représenter »}

C'est à cette démarche de « remplacement » que fait référence l'article de dictionnaire évoqué ci-dessus :

On notera qu'un certain nombre de linguistes préfèrent parler de "discours représenté » qu'utiliser l'appellation traditionnelle de « discours rapporté » (Fairclough 1988, Roulet 1999) qui reflète imparfaitement la diversité des phénomènes concernés.

Ainsi, pour Fairclough (1988), visant notamment l'incidence du contexte sur le sens des faits de RDA dans le discours médiatique, le rejet de « speech reporting » au profit de « discourse representation » se fonde-t-il ${ }^{11}$ sur l'inaptitude de « report » à faire place à la dimension interprétative de la représentation ${ }^{12}(c f$. point (b) ci-dessus).

9 Ce qui n'exclut pas, bien entendu, que cette dénomination ait été proposée bien antérieurement : Strauch (1974 : 41, 64) attribue à Jespersen (1924: 299 sq.) la paternité de « represented speech » et signale sa reprise par exemple en 1953 dans une thèse intitulée : « Represented discourse in the novels of François Mauriac » (A.G. Landry, 1953, Washington).

10 Notons aussi, chez Marnette (2005), le remplacement de « reported speech » par presentation, pour sa « neutrality » relativement à representation perçu comme porteur d'une idée de «reproduction».

11 Outre la lecture qu'il fait de speech reporting comme renvoyant exclusivement à l'oral.

12 "I use "discourse representation" rather than the more familiar "speech reporting" because (a) writing as well as speech may be represented and (b) rather than a transparent "report" of what was said or writen, there is always a decision to interpret or represent in one way rather than another. » (1995a : 54) 
Pour Roulet (1999) ${ }^{13}$, dans un texte non focalisé sur le champ du discours représenté/rapporté, mais dans le cadre d'une approche globale du discours, le choix du terme est donné, dans une note, comme "suivant l'usage de Fairclough », mais la justification donnée à la renomination est tout autre : non pas du côté (b) de la prise en compte de l'interprétation mais entièrement de celui (a) de l'extension du champ aux discours que " rapporter » se prête mal à inclure - discours non « énoncés antérieurement » ou « non formulés mais seulement désignés » ${ }^{14}$. De même, L. Rosier (2002 : 28), notant que certaines études de presse insistent sur la dimension paradoxalement fictive des discours rapportés, signalet-elle que Johansson (2000)

préfère à l'étiquette discours rapporté celle de discours représenté car le discours rapporté peut aussi être inventé. (idt)

\subsection{Inclure « rapporté » dans « représenté »}

Chez Nølke et al. (2004), l'appellation « discours représenté », présente dès le titre du chapitre consacré au champ, est donnée comme « terme générique pour tous les types de discours rapporté » (p. 57). Ce choix terminologique ne correspond pas ici, comme dans les cas précédents, à une critique spécifique, de type (a) ou (b), du terme « rapporté » : celui-ci est d'ailleurs conservé pour dénommer les formes particulières comme « Discours Direct Rapporté » ou « Discours Indirect Rapporté $»^{15}$. Il relève du cadre théorique d'ensemble de la Scapoline, héritière de la polyphonie linguistique de Ducrot : à la conception d'un statut réflexif de l'énonciation ( $c f$. " dès qu'on parle on parle de sa parole ») répondent, dans ce cadre, l'appréhension du « dire comme une représentation théâtrale » où « la parole est donnée à différents personnages » par un locuteur « metteur en scène » et celle du sens comme « la représentation qu'il donne de son énonciation ${ }^{16}$.

Aussi l'appellation de « discours représenté » n'y a-t-elle pas valeur de remplacement critique du terme " rapporté » mais celle d'une inscription des formes de ce champ dans le large concert polyphonique de la « représentation énonciative »,

13 p. 228, note 69 (renvoie au même texte que la référence (citée ci-dessus) (1999) ; $c f$. aussi Roulet (1997).

$14 C f$. par exemple : Il raconte son enfance : discours « désigné » sans « formulation » du message (comme le serait : Il raconte qu'il est né à..., en..., qu’il a eu...).

15 Cf. par exemple les deux tableaux synoptiques, p. 61, p. 67 et Glossaire p. 176.

16 Cf. notamment Ducrot $(1980,1984)$, l'article « Polyphonie » signé de H. Nølke in Charaudeau et al. (2002) et des éléments d'analyse critique in Authier-Revuz (1995/2012 : 55 sq., 79 sq.) 
aux côtés des formes de négation, présupposition, connecteurs, etc. ${ }^{17}$. La dimension de la « représentation », transverse à l'ensemble des fait de polyphonie - aux discours rapportés comme aux " points de vue » portés par les « êtres discursifs » mis en jeu dans la négation, etc. -, n’est pas spécifique au discours rapporté, qui apparaît comme un «type particulier » de polyphonie (et donc de représentation) en ce qu'il « représente » du discours plutôt qu'un « point de vue ${ }^{18}$ Notons qu'il y a incompatibilité entre cette notion « étendue » de la représentation (solidaire de la conception « représentative » du sens et de l'énonciation) et l'opposition hétérogénéité représentée vs constitutive (ou " présente ») (cf. Authier-Revuz 1980, 2012), sous-jacente à mon traitement de la RDA (voir plus loin partie V)..

Chez Rabatel ${ }^{19}$ également, la dénomination de " discours représenté », plus qu'une critique du terme « rapporté », répond à un élargissement de la problématique : pour lui,

l'articulation entre PDV et discours rapporté [entraîne] une redéfinition de la notion de discours rapporté comme discours représenté (Rabatel 2008b : 354).

$\mathrm{Au}$ cœur de cette démarche, un ancrage « perceptuel » de la notion de « point de vue » (PDV) comme « mixte de pensées, de perception et de paroles » et la conception d'un « axe pré-réflexivité/réflexivité » sur lequel se déploient, en continuum, les « comptes-rendus perceptuels de pensée et de parole » (ibid. : $353)^{20}$. Par rapport à l'acception classique du discours rapporté, il s'agit, " sous une optique moins strictement grammairienne [...] et davantage pragmatique et philosophique » (ibid. : 355), d'envisager, avec le « discours représenté »,

un ensemble qui comprend d'une part les discours rapportés grammaticalisés, auxquels on associe des formes de rapport/représentation moins grammaticalisées ( $c f$. les modalisations autonymiques d'Authier-Revuz, les formes mixtes de Rosier, d'autre part les différents

17 Ainsi, à la caractérisation de la négation comme « phénomène linguistique polyphonique par excellence » (Nølke et al.) fait écho, en introduction au chapitre « Discours représenté » : le « discours rapporté sera évidemment le phénomène textuel polyphonique par excellence» (p. 57).

18 «En termes polyphoniques, le Discours représenté est un type particulier de polyphonie externe [...] caractérisé par le fait de représenter le discours - ou plus précisément l'énoncé d'autrui et non seulement un PDV étranger. » (Nølke et al. 2004 : 58 sq.)

19 Dans la partie I du tome II de Homo narrans (2008b), intitulée « Points de vue, discours représentés, dialogisme et (pré)réflexivité », qui regroupe des articles parus sur ce thème entre 2001 et 2008, précédés d'une introduction (p. 345 sq.) d'où sont extraits, sauf indication contraire, les passages cités.

20 Le parcours de ce continuum donnant lieu à une riche exploration de frontières indécises entre DIL, DDL, monologue intérieur, PDV représentés, « infraverbalisés » ou « embryonnaires »... 
PDV plus ou moins pré-réflexifs, cet ensemble s'inscrivant lui-même dans le vaste domaine du dialogisme). (ibid. : 355$)^{21}$

\section{Du « Discours Rapporté » à la « Représentation de Discours Autre " (RDA)}

Le choix de l'appellation « Représentation de Discours Autre » participe des deux démarches évoquées ci-dessus (en 2).

Il repose en effet - on l'a vu dans le premier parcours du champ, en 1. ci-dessus - négativement (comme Fairclough ou Roulet) sur les raisons de ne pas conserver, en dépit de l'usage établi, le trop inadéquat « discours rapporté »; et ces raisons relèvent des deux plans distingués (a) et (b) :

- celui, (b), où « représenter », en évitant les connotations d'objectivité transparente attachées au « rapport » fait place au caractère interprétatif de l'opération ;

- celui, (a), où « représenter », par son caractère général, évite des restrictions de l'extension du champ auxquelles se prête le sens de « rapporter ». Il s'agit, d'une part, au plan du référent représenté (l'acte $\boldsymbol{a}^{\mathbf{0}}$ ), d'inclure - au delà du modèle « $\boldsymbol{l}$ a dit... » tous les faits de discours futurs, niés, interrogés, imaginés..., n’ayant pas « eu lieu ». D’autre part, d'inclure, parmi les discours représentés, à côté de ceux que le Dire représente au titre de son objet (« ce dont il parle ») comme il dit $P$, il dit « $X » . .$. , ceux qu'il représente au titre de sa source ( « ce d'après quoi il parle »), comme d'après ce qu'il dit $P$, «X » comme il dit, qui interviennent dans le champ de la RDA comme Modalisation du Dire par discours autre (MDA) ${ }^{22}$. L'extension de la RDA inclut donc celle, usuelle, du « discours rapporté ».

Le choix de « Représentation de Discours Autre » relève aussi du choix foncier, positif, de marquer l'appartenance du champ à l'espace, plus vaste, du métalangagier. Nous avons évoqué ci-dessus ce même mouvement d'inscription du champ dans une problématique élargie, mais dans des directions autres : celle de l'énonciation comme représentation dans la perspective polyphonique (Scapoline) et celle d'une réflexivité étendue aux points de vue et à la perception (Rabatel). La démarche suivie ici envisage le « discours rapporté », renommé - et redessiné -

21 La structuration du champ de la RDA que je propose dans cet ouvrage ( $c f$. notamment chap. 9) ne passe pas par cette distinction « grammaticalisé/moins grammaticalisé ».

22 Cf. ci-dessus chap. 1.2.4, p. 32 et ci-dessous chap. 3. 
en RDA, comme secteur de la pratique métalangagière, au sens où J. Rey-Debove (1978) la circonscrit et la balise. C'est comme partie prenante de cet étage du langage - langue et discours - permettant de revenir sur le langage - langue ou discours - que la RDA est envisagée.

Le secteur spécifique de la RDA sera ainsi appréhendé par rapport à ses « voisins en métalangage » (voir tableau I), ce qui, sur la base de ce qui les réunit - les opérations transverses à l'ensemble, de la catégorisation, de la paraphrase et de l'autonymie, envisagées partie III. - permet d'y dégager ce qui est le propre de la représentation du discours (et non de la langue, $c f$. chap. 1.2.2), et du discours autre $^{23}$ (et non du « même » de l'ARD, $c f$. chap. 1.2.3).

23 « Discours autre » n'est pas, on le rappelle ( $c f$. ci-dessus chap. 1.2.3, p. 17 et chap. 2.2.1, p. 53), synonyme de « discours d'autrui ». ; le « discours autre » inclut le « discours de soi » dès lors que celui-ci ne coïncide pas avec le « discours en train de se faire». 